\title{
Non-intubated thoracoscopic surgery (NITS) is a safe alternative of one lung ventilation (OLV) in patients undergoing video assisted lobectomy: a case control, non-inferiority, pilot study
}

\author{
Z. Szabó ${ }^{1}$, T. Tanczos ${ }^{1}$, G. Lebak ${ }^{1}$, F. Hawchar ${ }^{1}$, J. Furak², Z. Molnar' \\ University of Szeged, Faculty of Medicine, Department of Anaesthesiology and Intensive Therapy, \\ University of Szeged, Faculty of Medicine, Department of Surgery \\ ${ }^{1}$ Department of Anaesthesiology and Intensive Therapy, University of Szeged, Faculty of Medicine - Szeged (Hungary), ${ }^{2}$ Department \\ of Surgery, University of Szeged, Faculty of Medicine - Szeged (Hungary)
}

\section{Background and Goal of Study:}

Video-assisted thoracoscopic surgery (VATS) has become a widely accepted approach worldwide. Conventionally it is performed with lung isolation and mechanical ventilation. Non-intubated thoracoscopic surgery is a novel approach (1), with theoretical benefits of the avoidance of endotracheal intubation and positive pressure ventilation. However, little is known about its pathophysiological effects. Therefore, our goal was to compare the effects of NITS versus OLV on systemic inflammation, lung function and gas exchange during and after lobectomy.

Materials and Methods: In a prospective observational case-control, non-inferiority study patients undergoing NITS were compared to patients managed with OLV. The primary end points were postoperative procalcitonin (PCT) and C- reactive protein (CRP) levels. Blood samples were drawn preoperatively (T0) and postoperatively at T12, T24, T48. Secondary outcomes were: postoperative lung function (LFT); arterial blood gases (ABG) determined pH, pO2 and pCO2, lactate; white blood cell counts (WBC), chest drain removal and length of hospital stay (LOS).

\section{Results:}

Over a period of 12 months 61 patients were involved in our study, 36 undergone NITS and 25 patients had OLV. There was no difference between the two groups in demographics (age, gender, type of tumor) and in length of surgery. CRP was significantly lower at T48 in the NITS-group: median = 56.8 (IQR=32.7-75.8) vs. $126.4(71.9-143.5) \mathrm{mg} / \mathrm{l}, \mathrm{p}<0.01$. There was no significant difference in PCT levels $(\mathrm{ng} / \mathrm{ml})$ at each assessment point: T0: median $=0.06$ (IQR: 0.06-0.06) vs. 0.06 (0.06-0.06), T12: $0.09(0.06-0.17) \mathrm{ng} / \mathrm{ml}$ vs. 0.09 (0.06-0.12), T24: $0.11(0.06-0.20)$ vs. $0.11(0.06-0.18)$, T48:0.1(0.06-0.16) vs. 0.09(0.070.14 ); respiratory function, $A B G$ and WBC. Chest drain removal (median=2[IQR=2-4] vs. 3[3-4] days, $p=0.025$ ).
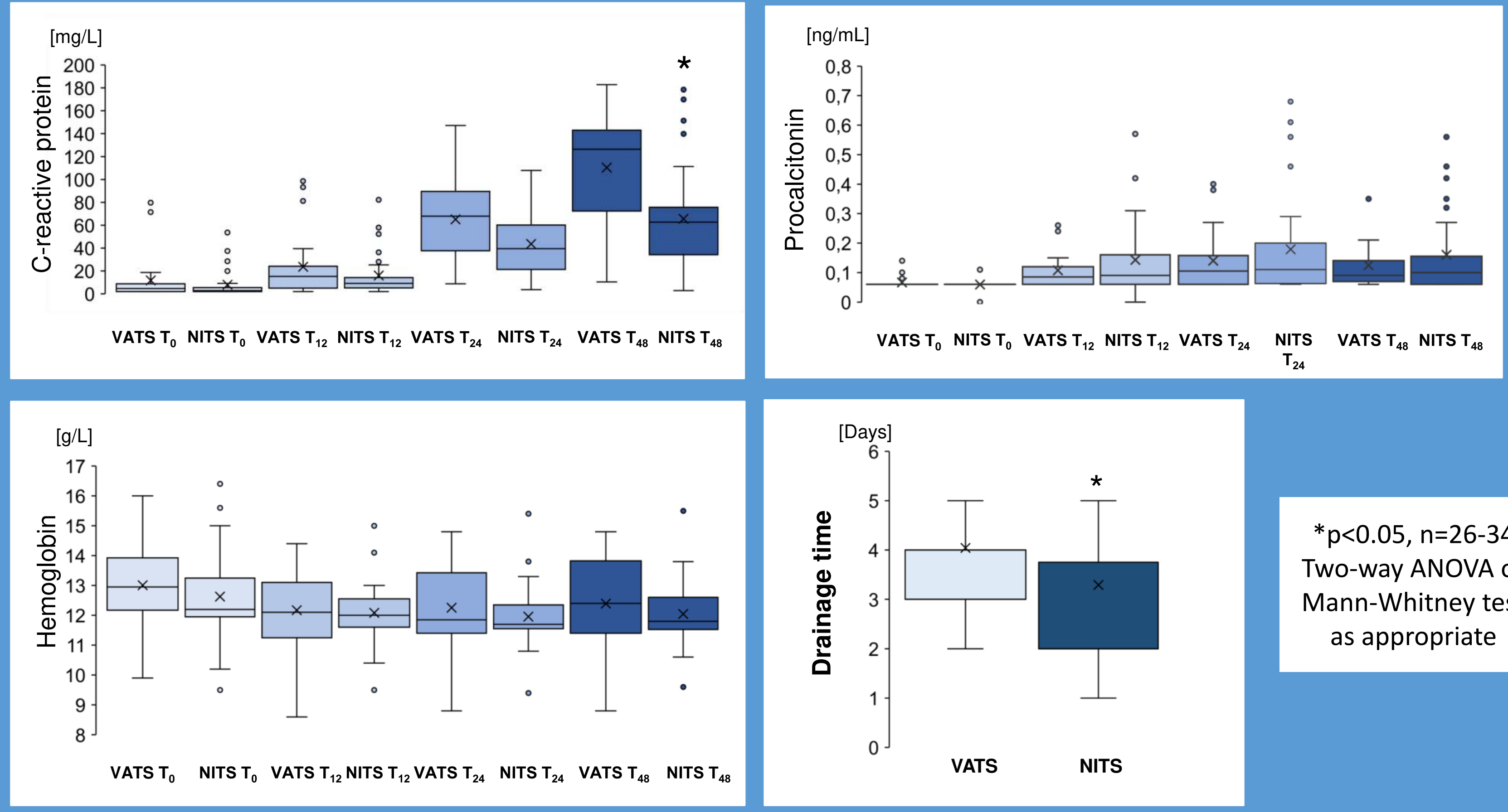

${ }^{*} p<0.05, n=26-34$ Two-way ANOVA or Mann-Whitney test as appropriate

\section{Conclusion(s):}

To our best knowledge this is the first study suggesting that NITS causes similar surgical stress caused inflammatory response (or even better as far as CRP is concerned), postoperative oxygenation and ventilation as compared to OLV. Therefore, it seems to be at least a non-inferior alternative of OLV and may also provide benefits for patients undergoing lobectomy such as shortened need for chest drains and earlier discharge. 